\title{
Lipoflavonoid Benefits in Patients with Tinnitus
}

\author{
Lori E. Lonczak \\ Lori Lonczak Consulting, LLC, Fair Haven, USA \\ Email: lorilonczak@gmail.com
}

How to cite this paper: Lonczak, L.E. (2021) Lipoflavonoid Benefits in Patients with Tinnitus. International Journal of Otolaryngology and Head \& Neck Surgery, 10, 147-157.

https://doi.org/10.4236/ijohns.2021.103015

Received: March 6, 2021

Accepted: May 7, 2021

Published: May 10, 2021

Copyright (c) 2021 by author(s) and Scientific Research Publishing Inc. This work is licensed under the Creative Commons Attribution International License (CC BY 4.0).

http://creativecommons.org/licenses/by/4.0/ (c) (i) Open Access

\begin{abstract}
Background: Otolaryngologists are regularly presented with patients experiencing tinnitus who are seeking treatment to help with resuming active and productive lives. Lemon bioflavonoid complexes are known to be safe nutritional supplements; however, data on patient satisfaction are limited. Objectives: The primary objectives were to better understand symptom severity reduction, symptom improvement, and patient satisfaction when lemon bioflavonoid complexes are taken at the recommended dose and duration of treatment. Methods: The SILENT experience study was conducted from January 2017 to January 2018, with 504 otolaryngologists from six states, identified from a purchased list. Each physician provided a free, 500-caplet sample of Lipo-Flavonoid ${ }^{\oplus}$, a lemon bioflavonoid complex, to three patients who experienced tinnitus and completed a survey detailing patient demographics and symptoms. In total, 719 patients received the sample; 51 patients followed through and completed a baseline symptom assessment and five surveys over 10 weeks. IRB approval was deemed unnecessary and the study was overseen by a practicing otolaryngologist. Statistical analysis was conducted on the reduction of symptom severity and symptom improvement over 10-weeks. Results: Bothersomeness of ringing or other noises in the ear(s) was reduced by $32 \%$ and patients reported a $55 \%$ improvement of all symptoms, which correlated with product satisfaction. Patient satisfaction scores were strong: $33 \%$ extremely satisfied, $49 \%$ somewhat satisfied, and $18 \%$ not satisfied. Compliance was high, with $86 \%$ of participants taking the product as directed for 60 days. The top reasons for physician recommendations (selected by $>30 \%$ ) were positive clinical experience $(71 \%)$, good safety profile (66\%), supported by data for over 50 years (37\%), and efficacy (35\%). Implications for Practice: These data support the use of this lemon bioflavonoid complex for symptoms of tinnitus, for 60 days (and beyond for those who derive benefit) to provide the best chance of relief. For clinicians looking for a safe and beneficial intervention for patients, lemon bioflavonoid
\end{abstract}


complexes offer a good first-line option.

\section{Keywords}

Lipoflavonoid, Bioflavonoid, Tinnitus, Ringing

\section{Introduction}

Tinnitus is largely self-reported and is subjective in nature. There is no standard definition of tinnitus, but it is generally deemed to be the perception of sound when there is no audible source. This "phantom" sound can be heard in one or both ears. Patients are typically considered to have tinnitus if they chronically hear noises, with some studies defining it as enduring for three or more months [1], and recent guidelines defining tinnitus as six months or more of persistent symptoms [2].

Tinnitus is recognized as debilitating, affecting a patient's overall health and quality of life. Tinnitus patients are at risk for anxiety and depression, with $48 \%$ - $60 \%$ of patients reporting depression [2]. It is also linked to a variety of risk factors and co-morbid conditions such as autoimmune disease, hypertension, diabetes, dyslipidemia, and arthritis [3].

As a result, tinnitus is a serious economic and social burden in the U.S. Not only does it negatively impact workplace productivity, but it is also a major source of disability and health care costs for men and women.

Patients with tinnitus may have problems with social interactions in addition to other lifestyle issues. They often experience difficulty with work and bouts of insomnia [4]. The impact of tinnitus is considerable because it affects hearing, concentration, and sleep as well as other social activities.

Table 1 details data from Intercontinental Medical Statistics (IMS) medical claims data in 2015; showing 369,220 patients were treated for tinnitus, with $20 \%$ being newly diagnosed. Patients aged 55 - 64 represented $27 \%$ of the total, while patients aged $65+$ represented $36 \%$, providing evidence that prevalence increases with age [5].

Despite the relatively low numbers of patients being treated for tinnitus by healthcare professionals, it is not an uncommon condition, particularly among older adults.

The prevalence of tinnitus is estimated at 25.3\%. A 1996 National Health Interview Survey estimated that 35 - 50 million adults have tinnitus, with 12 million seeking medical help and $2-3$ million experiencing severely debilitating symptoms [6]. Historically, men were more likely to experience tinnitus than women because there was a strong correlation with exposure to occupational noise [6]. Interestingly, 2015 IMS medical claims data show that $55 \%$ of patients treated for tinnitus were women. Of newly diagnosed patients, women accounted for $57 \%$, with men representing only $43 \%$ of the total [5]. 
Table 1. Total and newly diagnosed patients with tinnitus.

\begin{tabular}{cccc}
\hline Age & \# of Patients & \% of Total Patients & \% Newly Diagnosed \\
\hline $18-24$ & 11,634 & $3 \%$ & $5 \%$ \\
$25-34$ & 21,159 & $6 \%$ & $7 \%$ \\
$35-44$ & 35,088 & $9 \%$ & $11 \%$ \\
$45-54$ & 68,466 & $19 \%$ & $18 \%$ \\
$55-64$ & 98,362 & $27 \%$ & $26 \%$ \\
$65+$ & 134,510 & $36 \%$ & $33 \%$ \\
TOTAL & 369,220 & $100 \%$ & $100 \%$ \\
\hline
\end{tabular}

[IMS medical claims, 2015].

Recent guidelines published by the American Association of Otolaryngologists evaluated currently available treatment options for tinnitus and issued recommendations for the following non-pharmacologic treatments: hearing aids/amplification, sound therapy, and cognitive behavioral therapy. No pharmacologic treatments have been FDA approved for tinnitus, and prescription drugs (antidepressants, anticonvulsants, anxiolytics), ginkgo biloba, melatonin, zinc, and other supplements are not recommended [2].

In September 2019, a tinnitus guideline advisory meeting was held with key opinion leaders to develop consensus recommendations to fill in gaps not addressed by existing clinical practice guidelines. The advisory group reported that bioflavonoids are one of the most commonly used supplements for managing tinnitus symptoms. Furthermore, lemon bioflavonoids (eriodictyol glycoside) and a mix of Vitamins (B1, B2, B6, B12, C) are thought to help inner ear circulation and block histamines. The guideline advisory emphasized that appropriate medical and audiological evaluation, along with determining a clear understanding of tinnitus management goals and a defined trial period, are important to consider when trying supplements.

Lemon bioflavonoid complexes containing eriodictyol glycoside have been used extensively for decades as part of a multimodal approach for the treatment of tinnitus [7]. The efficacy of such complexes was first documented by Dr. Henry L. Williams, of the Mayo Clinic in the 1960s in a series of papers evaluating their use in the treatment of patients with symptoms consistent with Meniere's Disease. Based on those findings and subsequent work, he speculated that eriodictyol glycoside improves microcirculation in the inner ear, helps prevent abnormal accumulation of fatty deposits, and possibly blocks histamine production. More than 50 years of clinical data support the notion that lemon bioflavonoid complexes provide symptom improvement and relief from vertigo and tinnitus [7]. These data also suggest that it is likely that supplementation for at least two months may be required for symptom relief [8]. Today, otolaryngolo- 
gists and audiologists regularly employ supplementation with lemon bioflavonoid complexes as primary or adjunctive therapy in cases where ringing or other noises in the ear(s) are involved.

\section{Objectives}

A product trial program was initiated to assess patient experience and satisfaction with lemon bioflavonoid complexes used for 60 days as well as to learn physicians' reasons for dispensing the product. The objectives of the study were to:

- Learn why otolaryngologists recommend the product to patients;

- Collect data on symptom severity reduction, symptom improvement, and patient satisfaction when lemon bioflavonoid complex is taken at the recommended dose for 60 days;

- To better understand patient benefits, patient satisfaction, and likelihood of success with lemon bioflavonoid complexes.

\section{Methods}

Physicians were identified using a list of 1006 practicing otolaryngologists from six states (NY, PA, FL, OH, TX, CA) purchased from IQVIA and recruited by phone to participate in the study. Of the otolaryngologists on the list, 504 participated. There were no additional selection criteria.

Participating physicians were given three bottles of 500 caplet samples of Lipo-Flavonoid $^{\circledR}$, a lemon bioflavonoid complex to dispense to three patients for symptoms of tinnitus. The study was funded by and the product was provided by Clarion Brands, makers of the Lipo-Flavonoid ${ }^{\circ}$ commercial product. The ingredients are shown in Table 2. These physicians were not compensated, and they provided samples to 719 patients.

Table 2. Commercial product ingredients (https://lipoflavonoid.com).

Ingredients in Lemon Bioflavonoid Complex
Eriodictyol glycoside (lemon citrus bioflavonoid)
Vitamin C (as ascorbic acid)
Vitamin B-1 (thiamine mononitrate)
Vitamin B-2 (riboflavin)
Niacin (niacinamide)
Vitamin B-6 (pyridoxine HCl)
Vitamin B-12 (cyanocobalamin)
Pantothenic acid (as calcium pantothenate)
Choline Bitartrate
Inositol


The otolaryngologists participating in this study (open-label, not placebocontrolled) were asked to complete a short survey for each selected patient on a prepaid confidential postcard that included patient demographics and reasons for dispensing the sample (from a pre-specified list of reasons). Patients were asked to self-report on symptoms and product satisfaction by completing a baseline assessment (survey 0 ) and five additional surveys, once every 2 weeks, over a 10 -week period as shown in Table 3. Patients were provided a pack of six surveys on confidential (results not visible) prepaid postcards. In total, 51 patients (out of the 719 who received the product) completed the surveys.

Table 3. Patient questions and possible responses.

\begin{tabular}{cc}
\hline Question & Possible Responses \\
\hline $\begin{array}{c}\text { Do you experience ringing (or other noises) } \\
\text { in your ears? }\end{array}$ & (Yes/No) \\
\hline $\begin{array}{c}\text { Do you experience dizziness? } \\
\text { How long have you been suffering } \\
\text { from these symptoms? }\end{array}$ & 0 - 2 months \\
\hline
\end{tabular}

Not bothersome 123

As of today, please rate how bothersome the Somewhat bothersome 4567 ringing (or other noises) in your ears is when it's most bothersome?

Extremely bothersome 8910

Not applicable

Not bothersome 123

As of today, please rate how bothersome the Somewhat bothersome 4567 dizziness is when it's most bothersome?

Extremely bothersome 8910

Not applicable

\begin{tabular}{cc}
\hline $\begin{array}{c}\text { As of today, please describe what time(s) } \\
\text { of day or night your symptoms are } \\
\text { most bothersome? }\end{array}$ & Open text response \\
$\begin{array}{c}\text { Except for this bottle, have you ever taken } \\
\text { Lipo-Flavonoid Plus before? }\end{array}$ & Yes \\
& No \\
$\begin{array}{c}\text { If you took Lipo-Flavonoid Plus in the past, } \\
\text { were you satisfied with symptom relief? }\end{array}$ & No \\
& Never took before \\
Is using a product that is \#1 doctor \\
recommended important to you?
\end{tabular}


Data from physician and patient response cards were compiled in an Excel spreadsheet as cards were received then checked by a second analyst. Answers to survey questions were put into a report of findings by the author and reviewed by the study chair (Dr. Jay Horowitz, MD, FACS, Chief of the Division of Otolaryngology, Head \& Neck Surgery at St. Peter's University Hospital, Clinical Associate Professor of Surgery/Otolaryngology at Rutgers Medical School and practicing otolaryngologist at ENT and Allergy Associates in New Jersey).

Individual responses to all questions were collected, organized, and sent to RAS Statistics for statistical analysis.

The statistical analysis was conducted on the mean reduction in the severity of ringing and/or noise in the ear(s) from baseline as well as for patient-reported scores on improvement of all symptoms, including ringing and/or other noise in the ear(s) and dizziness. Calculations were done in $\mathrm{R}$ Version 3.4.2 and verified in Excel.

- Patient-reported scores on ringing (or other noises) in the ears: Scores could range from 1 to 10 with higher numbers indicating more bothersome symptoms. The scores were first collected on survey 0 (baseline) and then subsequently on surveys 1 - 5 .

- Patient-reported scores on improvement of symptoms: Scores could range from 1 to 10 with higher numbers indicating greater improvement. The scores were collected on surveys $1-5$. Note that since the scores are themselves ratings of improvement, there is no baseline to which to compare subsequent scores.

\section{Results}

\subsection{Otolaryngologist Respondents}

The study was conducted from January 2017-January 2018. A total of 504 otolaryngologists participated, and they reported on baseline symptoms for 719 patients as detailed in Figure 1. Ninety percent of patients had ringing in their ears, while 54\% experienced other noises in their ears like buzzing and clicking and $36 \%$ had vertigo. Interestingly, 58\% of patients experienced hearing loss, and of that subset, $57 \%$ had mixed hearing loss, $15 \%$ sensorineural and $28 \%$ conductive.

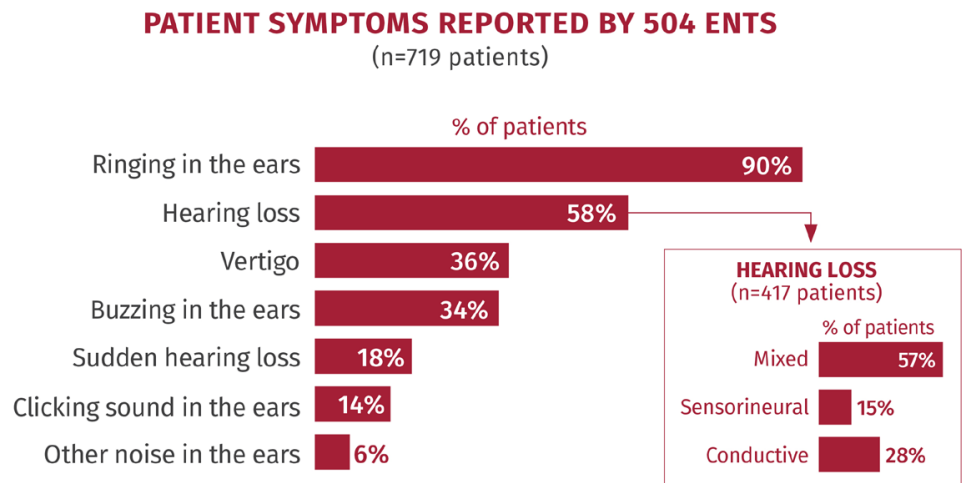

Figure 1. Patient symptoms at baseline. 
ENTs also selected their top reason(s) for recommending the supplement to each patient from a pre-specified list of reasons. This portion of the information was completed on 713 of the 719 submitted cards. Physicians reported the following reasons for dispensing the sample: positive clinical experience (71\%), good safety profile (66\%), supported by clinical data for over 50 years $(37 \%)$, efficacy $(35 \%)$, colleague or peer experience $(20 \%)$, formulation contains the lemon bioflavonoid, eriodictyol glycoside (20\%), and other (15\%).

\subsection{Patient Respondents}

Responses to the surveys were collected from 51 patients; each received one 500-caplet sample and six survey cards. Patients reported on their symptoms and product satisfaction on a baseline assessment (survey 0); they then completed five surveys over a 10-week period (surveys 1 - 5). Twelve patients completed the baseline survey and no additional surveys so these data were excluded. The following baseline data were captured:

- $61 \%$ of patients were men and $39 \%$ were women $(n=51)$

- $87 \%$ of patients enrolled were 51 years of age or older $(n=51)$

- $74 \%$ were Caucasian, 11\% Hispanic, 8\% Asian, 5\% Middle Eastern, and 2\% African American $(\mathrm{n}=51)$

- Patient length of time with symptoms varied: 51\% (12 months+), 28\% (3-12 months), and $21 \%(0-2$ months) $(\mathrm{n}=46)$

- Time of day patients experienced symptoms: 50\% when quiet (early morning, evening), $36 \%$ constant, and $14 \%$ daytime $(n=44)$

- Baseline symptom severity was 7.8 out of 10 on average (" 10 ” was considered "extremely bothersome" and "0" was considered "not bothersome"), with $64 \%$ rating $8-10,34 \%$ rating $4-7$, and $2 \%$ rating $1-3$ ( $\mathrm{n}=51$ ringing/noises; $\mathrm{n}=24$ dizziness)

\subsection{Patient Findings}

At baseline, patients reported a 7.8 (out of 10) average severity of ringing or other noises in the ears (when most bothersome), with 10 being the most bothersome. Every two weeks, the patient respondents completed the same survey and over time, a severity reduction of $32 \%$ was seen, with the final survey showing an average score of 5.3 out of 10 . Individual survey results over time are shown in Figure 2.

With regard to the scores for ringing/noises in the ears, the mean reductions from baseline (survey 0 ) are statistically significant for every follow up survey. For each of surveys 1 - 5, the 2 -sided p-value is less than 0.001 . This remains true even when the four missing values (one on survey 3 , two on survey 4 , and one on survey 5) are replaced with the maximum score of 10.

Patients also reported symptom improvement over time on the surveys. Overall symptom improvement at survey 1 (vs. survey 0 ) was $34 \%$ and by survey 5 , the overall aggregate symptom improvement was 55\%. With regard to the scores 


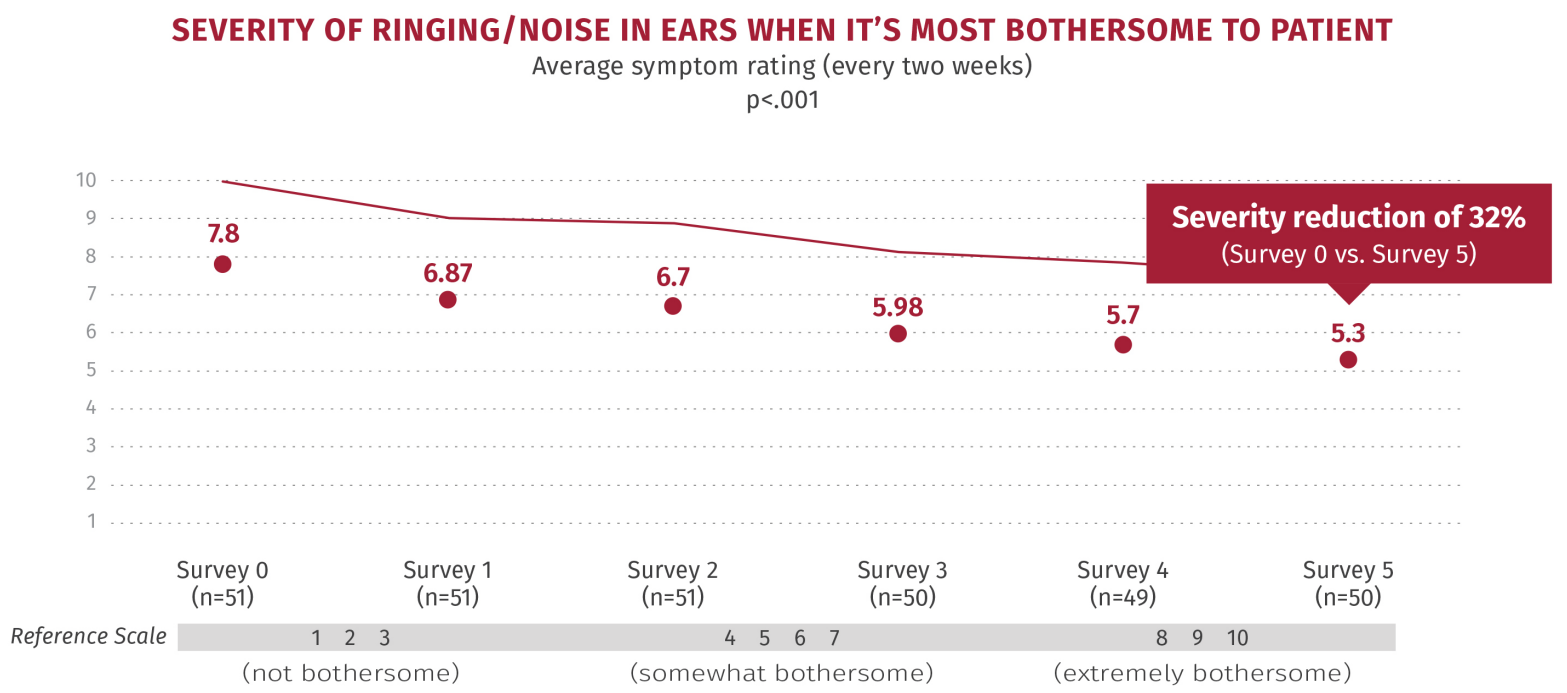

Figure 2. Severity of ringing/noise in ears when most bothersome to patient.

for improvement of symptoms, the estimated slope of the population regression line is approximately +0.5 , indicating a highly statistically significant (2-sided p-value $<0.001)$ increasing trend in symptom improvements.

Overall patient satisfaction with the lemon bioflavonoid complex was correlated with symptom improvements as shown in Figure 3. Altogether, $82 \%$ of patients were "extremely" satisfied or "somewhat" satisfied, with $18 \%$ getting little-to-no relief.

\section{Discussion}

Tinnitus is a common condition in both men and women, with over 50 million people in the United States have reported experiencing it, resulting in an estimated prevalence of $10 \%-15 \%$ in adults [6]. Historically, the condition was primarily seen in men; however, recent data show a trend toward increasing incidence in women [1].

Despite the high prevalence and significant impact on quality of life, there is no known cure for most types of tinnitus, and there are currently no FDA-approved treatments.

Data from the SILENT study demonstrate that supplementation with the lemon bioflavonoid complex examined in this study can ease the burden of tinnitus, allowing patients to live more comfortable, productive lives. Some of the key findings from the study are listed below:

- Severity of ringing/noise in the ear(s) when it is most bothersome to patients was rated 7.8 out of 10 at baseline, and it was reduced by $32 \%$ over the 10-week study period;

- Patient symptom improvement overall; $55 \%$ over the 10 -week study period;

- Patient satisfaction with the lemon bioflavonoid complex was high, with $33 \%$ of participants extremely satisfied, $49 \%$ somewhat satisfied, and $18 \%$ not satisfied; 


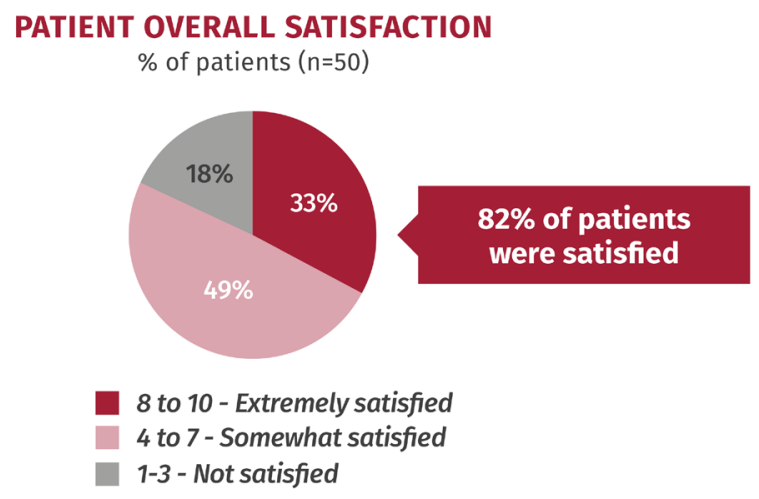

Figure 3. Overall patient satisfaction.

- Most patients reported being compliant, took two caplets three times a day, and remained on the lemon bioflavonoid complex for 60 days;

- Reduction in symptom severity was correlated with satisfaction.

As this was a real-world study, there are limitations. The analysis relied on reporting from patients who were instructed to complete a baseline survey plus one survey every two weeks for a total of six surveys over a 10-week period. Additionally, patients had to take two caplets three times daily for 60 days, so it is likely that only the most compliant and motivated patients participated. Out of 719 patients who received the sample and surveys, just 51 completed all six surveys. Twelve patients completed the baseline survey and did not follow up with the remaining five surveys therefore these baseline data were excluded from the analysis. Additionally, some questions were left unanswered by responding patients. Interestingly, the severity of symptoms at baseline for this group of responding patients was high, with an average self-reported score of 7.8 out of 10 (with 10 being the most bothersome). Further research in a controlled research setting is needed to improve the body of evidence and identify types of patients who may be more likely to benefit from supplementation.

\section{Implications for Practice}

The SILENT experience study collected baseline information from hundreds of otolaryngologists and data from 51 tinnitus patients; it included patient feedback on severity of symptoms and symptom improvement over time while taking the lemon bioflavonoid complex at the proper dose over a 60 day period. Otolaryngologists are regularly presented with patients experiencing tinnitus who are seeking treatment to help them resume active and productive lives.

The specific lemon bioflavonoid complex described in the methods section is known to be a safe nutritional supplement. However, until now, patient benefit and satisfaction data have been limited. Otolaryngologists participating in the SILENT open-label patient experience study reported that a positive clinical experience and good safety profiles were the top reasons for recommending this lemon bioflavonoid complex. Now, providers have more specific information on the likely benefits of this complex upon which to base their recommendations. 
This study report is especially timely since, in 2014, the American Academy of Otolaryngology guidelines offered relatively few options for treatment of tinnitus, including hearing aids/amplification, cognitive behavioral therapy, and sound therapy [2].

A 2019 tinnitus guideline advisory group recognized that bioflavonoids are one of the most commonly used supplements for managing tinnitus symptoms. It also emphasized that appropriate medical and audiological evaluation, along with determining a clear understanding of tinnitus management goals and a defined trial period, are important to consider when trying supplements.

Data from the SILENT study support the use of this lemon bioflavonoid complex in clinical practice for symptoms of tinnitus to provide the best chance of benefit. Of patients participating in the SILENT study, 67\% reported that they would continue taking the lemon bioflavonoid complex, $7 \%$ would not continue, and $26 \%$ did not know. Future research should focus on the identification of patients who are most likely to benefit from this lemon bioflavonoid complex. For clinicians looking for a safe and beneficial intervention for patients, this lemon bioflavonoid complex offers a good first-line option.

\section{Acknowledgements}

Jay B. Horowitz, MD, FACS, Chief of the Division of Otolaryngology, Head \& Neck Surgery at St. Peter's University Hospital, Clinical Associate Professor of Surgery/Otolaryngology at Rutgers Medical School, and practicing otolaryngologist at ENT and Allergy Associates in New Jersey, served as the study chair and provided guidance for the study and this article.

\section{Conflicts of Interest}

The author declares no conflicts of interest regarding the publication of this paper.

\section{References}

[1] Hoffman, H.J. and Reed, G.W. (2004) Epidemiology of Tinnitus. In: Snow Jr., J.B., Ed., Tinnitus: Theory and Management, B.C. Decker, Inc., Hamilton, Ontario, B.C., 6-41.

[2] Tunkel, D.E., Bauer, C.A., Sun, G.H., Rosenfeld, R.M., Chandrasekhar, S.S., et al. (2014) Clinical Practice Guideline: Tinnitus. Otolaryngology. Head and Neck Surgery, 151, S1-S40. https://doi.org/10.1177/0194599814545325

[3] Nondahl, D.M., Cruickshanks, K.J., Wiley, T.L., Klein, B.E., Klein, R., et al. (2010) The 10-Year Incidence of Tinnitus among Older Adults. International Journal of Audiology, 49, 580-585. https://doi.org/10.3109/14992021003753508

[4] Shargorodsky, J., Curhan, G.C. and Farwell, W.R. (2010) Prevalence and Characteristics of Tinnitus among US Adults. The American Journal of Medicine, 123, 711-718. https://doi.org/10.1016/j.amjmed.2010.02.015

[5] IMS Medical Claims Data (2005)

[6] National Institute of Deafness and Other Communicative Disorders (2012) National 
Health Interview Study Disability Supplement.

https://www.nidcd.nih.gov/health/statistics/prevalence-chronic-tinnitus-chart

[7] Mayo Clinic (2021) Menier's Disease.

https://www.mayoclinic.org/diseases-conditions/menieres-disease/symptoms-cause s/syc-20374910

[8] Shea Jr., J.J. (1965) The Treatment of Meniere's Disease. Journal of the Mississippi State Medical Association, 6, 411-414. 